\title{
EVOLUTION OF THE HILTON HEAD WORKSHOP RESEARCH COMMUNITY
}

\author{
Adam D. Troy, Guo-Qiang Zhang, and Mehran Mehregany \\ Department of Electrical Engineering and Computer Science \\ Case Western Reserve University \\ Cleveland, Ohio USA
}

\begin{abstract}
In this paper, we analyze the evolution of the Hilton Head Workshop research community using our new "sliding window" approach to network theory. To do so, we follow the Hilton Head Workshop from its inception, i.e., the first meeting in 1984, through 2004, the latest meeting. The Hilton Head Workshop research community and the field it represents have undergone many changes over the last 20 years, during which over 2000 researchers (i.e., paper authors) have published over 730 papers. Because the Workshop's inception date coincides closely with the emergence of MEMS as a distinct field and the Workshop's coverage is quite focused on MEMS, its evolution over the last 20 years provides insight into the evolution of the MEMS research community, particularly in North America. We find that the community has grown significantly since 1984, although it needs more time to become a fully "mature" community. Our analysis shows that the sliding window approach paints an accurate and realistic picture of the Hilton Head research community.
\end{abstract}

\section{INTRODUCTION}

Modern scientific research relies heavily on collaboration. This is due to many factors such as increasing researcher specialization, increasing expense of equipment and materials, as well as an increasing pressure to publish. At its core, collaboration is largely a social enterprise, requiring personal interaction between the participants, with varying levels of success. In order to begin collaborating, researchers must be introduced to one another in one form or another. New knowledge and ideas that have not yet been formally published often pass from one researcher to another in an informal fashion. Collaboration has been shown empirically and through studies to increase publication output [1]. The above reasons make "social networks" an ideal tool to study communities of researchers. In social networks, nodes represent people; a pair of nodes is connected by an edge if there is a relationship between the corresponding individuals, collaboration in this case. In the context of research communities, since publication data is archived, co-authorship can be used to identify collaboration. Social network studies of such communities can reveal a great deal about collaboration within the community, provide an indication of the maturity of the community, and reveal influential members of the community.

\section{BACKGROUND}

In this work, we study a research community as represented by its collaboration network. Collaboration (or co-authorship) networks are networks where nodes represent authors and an edge between two nodes indicates that the corresponding authors have been co-authors of a common paper. The notion of scientific collaboration networks was introduced by Price in 1965 [2]. A well-known example is the Erdös collaboration graph [3], from which Erdös numbers are derived.

Collaboration networks have seen recent interest from two different perspectives: social networks and complex networks. Sociologists, anthropologists, and psychologists often use social networks to study relationships between individuals in groups. Individuals are represented as nodes of a network and edges between them represent their relationship. Traditional studies of social networks can reveal information about how organizations are run and structured, the nature of human interaction and individual roles. Most social network research deals only with relatively small networks and does not include studies of network evolution, mostly due to the lack of availability of such data.

In the 1950s and 1960s, mathematicians Erdös and Rényi published their seminal papers establishing the study of random networks [4]. Random networks consist of a static collection of vertices where each pair of vertices is connected by an edge based on some fixed probability. Since that time, scientists have assumed that most networks of interest are governed by the theorems proposed by Erdös and Rényi. Recently however, scientists from various disciplines, physics most predominantly, have been studying large networks and have concluded that the old models do not accurately reflect the properties of most large networks. In order to address this deficiency, the study of complex networks has emerged as a thriving area of research. The most notable property of complex networks is the power law distribution of the degree of the nodes [5], where the degree of a node is the number of other nodes to which it is connected. Complex networks contain very few nodes with high degrees (referred to as hubs), but many nodes with very low degrees. Complex networks also display the "small world" property introduced by Watts and Strogatz [6]. Networks that display the small word property have a very short average distance between each pair of nodes. The distance between two nodes is calculated as the smallest number of links connecting the pair of nodes. If no path exists between two nodes, the distance between them is said to be infinity. This property is related in spirit to the popular notion of "six degrees of separation" [7], referring to the phenomenon that anyone in the world is only six acquaintances away. Complex networks occur almost everywhere. Examples include cell proteins, food chains, the topology of the internet, and the World Wide Web among many others.

Due to the recent interest in complex networks, several researchers have studied scientific collaboration networks. Collaboration networks are popular for complex network studies due to the availability of large data collections for analysis. Barabási [8] has studied the evolution of two scientific collaboration networks, mathematics and neuroscience, from a complex network perspective. In those studies, the network evolution is studied cumulatively by adding each year's activities to the network created by the activities of the previous years. Newman has similarly studied static collaboration networks ranging from biology, physics, mathematics [9], to computer science and biomedical research [10]. Newman's work focuses on comparison of various statistics and properties of the different networks rather than network evolution. Additional studies on various co-authorship networks appear in $[\mathbf{1 1 , 1 2}]$.

\section{METHODOLOGY}

In this section we present the definitions that are basic to our study. First, we discuss the networks we use to represent research 
ommunities. Next, we define the sliding window perspective for network evolution observation.

We represent research communities using relatively simple social networks. Each author in the community is represented as a node or vertex in the network. An edge exists between two vertices if and only if the authors represented by the nodes have collaborated as co-authors on a paper. A graph so constructed contains many nodes and edges. This graph is a good approximation of the relationships in the community that facilitate the production of research and publications. These relationships are also likely to provide the conduit for unobserved discussion and transfer of ideas within the community. Many other more complex representations are possible. For example, weighted edges could be used to represent the strength of the relationship between the co-authors, or multiple classes of links could be used to represent relationships between authors such as those who belong to the same institution or attended the same workshop. For a thorough review of networks and their properties see references $[13,14]$.

One of the unique and important aspects of our work is the use of something we refer to as the "sliding window perspective". The main idea behind the sliding window perspective is that, when analyzing the network representing a community at a specific instance in time, the network should be constructed using only the data from recent, time-relevant collaborations rather than the cumulative history of collaborations. The sliding window approach reveals a completely different kind of network topology. For example, when analyzing the network for a particular year, we may only use the previous several years of the network data for edge construction even if a twenty-year history of the community of interest is available. The main argument for this perspective is that, when the goal is to analyze a specific community as it actually exists at a specific instance in time, the collaborations which took place a significant period of time earlier (and have not been renewed recently) are not an indication of an existing connection in the network representing the community structure at the current time. The sliding window approach is crucial to studying the lifetime dynamics of a community network. Communities not only add members as time passes but also lose members as these members change field or retire, though these "exits" are not explicit. The sliding window perspective also provides a simple way of capturing this process. A related idea was used by Jin for modeling growth in social networks [15]. The sliding window is the basis upon which all of our observations are presented in this paper.

The size (i.e., how long back from the point of interest) of the window is highly dependent on the specific community of interest. The goal is to choose the window size that best approximates the network as it actually existed in that moment of time. We recommend choosing a window that is large enough to include all but the most extreme time-spans between successive collaborations for the same pair of authors. The idea behind this is that if two authors collaborate again after their initial collaboration a link must have still been present between them in the network to facilitate this new collaboration. If a window is chosen that is much smaller, links between collaborating authors will fall out of the estimated network when they likely still exist. If the window is chosen to be much larger, older relationships that no longer have an effect on the community or no longer exist will remain in the estimated network.

\section{ANALYSIS}

In order to get some intuition for how much the community has changed, Figs. 1 and 2 show the community initially after the 1984 meeting and lately after the 2004 meeting, respectively. The dramatic growth of the community is evident from these figures. In 1984, the separate clusters of the network are formed by those authors who are co-authors of a single common paper. By 2004, the community has become much more complex, displaying several large clusters along with many small, single-paper clusters. The large clusters of Fig. 2 are often sub-communities containing authors who all belong to a common institution such as a university, laboratory, or company.

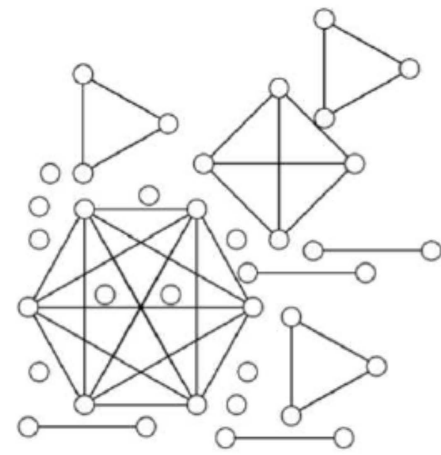

Figure 1. The Hilton Head community social network as it appeared after the 1984 meeting.

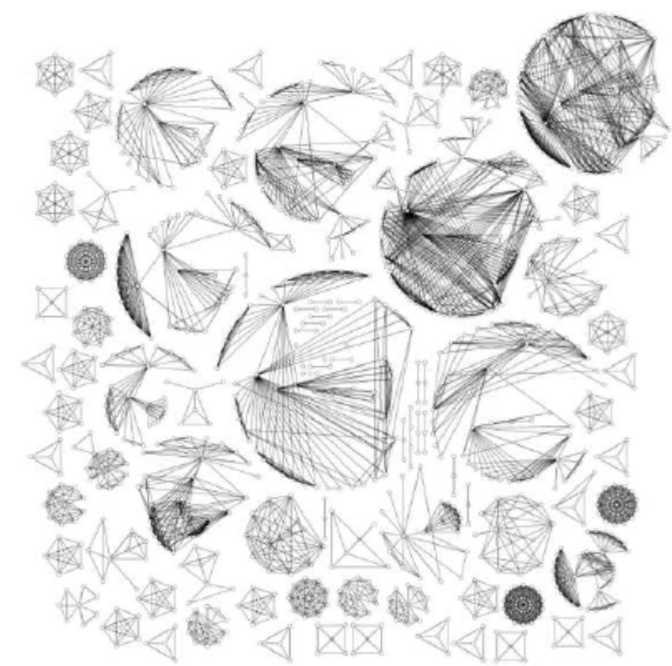

Figure 2. The Hilton Head community social network as it appeared after the 2004 meeting with a six-year sliding window.

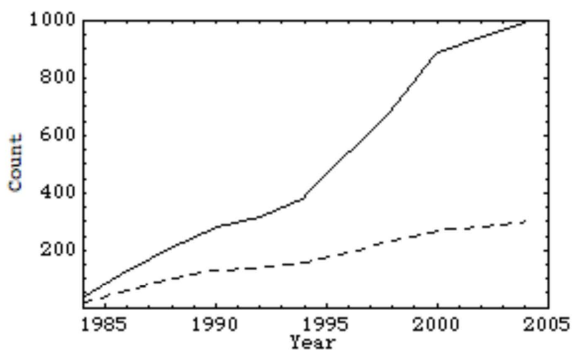

Figure 3. Evolution of number of authors (solid) and number of papers (dashed) for a six-year sliding window.

In this work, we use a sliding window of six-years. Therefore for each of the statistics discussed, the observations were gathered from a network constructed from the year shown and the preceding five years (other than the networks for 1984 and 1986, of course), for a total of three conferences per observation. Figure 3 shows the evolution of the number of authors and papers over the past twenty years of the community's life. In 1984, 37 researchers founded the 
community, while in 2004 the community had 989 researchers, a 26 -fold increase over 20 years! This increase is an indication not only of an increase in the average number of authors per paper, but also an increase in the diversity of the authors in the community, i.e., as opposed to the same small group of authors contributing papers year after year.

Figure 4 shows the evolution of the average number of papers per author, authors per paper, and collaborations per author. These statistics clearly portray the infancy of the MEMS discipline at the time of the founding of the Hilton Head Workshop, when authors often worked alone or in relatively small groups, whereas authors now have many collaborators, both on a per paper basis and overall. Figure 5 shows the evolution of the average component size for the 20-year lifetime of the community. In 1984, components were made up of only those authors which were coauthors on a single common paper, causing the average number of authors per paper (Fig. 4) and the average component size to be the same. This is in contrast to the current average component size, 10.6, which is significantly higher than the current average number of authors per paper of 4.5 (Fig. 4). This indicates that authors are collaborating with more researchers than would be explained by the increase in the average number of authors per paper and also that authors are collaborating with different groups of researchers for different papers. Additionally the growth of the number of components seems to have leveled off, implying that old components are merging and new authors are being introduced as part of existing components.

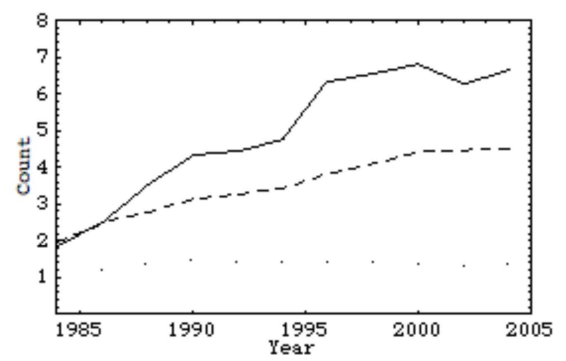

Figure 4. Evolution of average number of collaborations per author (solid), average number of authors per paper (dashed), and average number of papers per author (dotted) for a six-year sliding window.

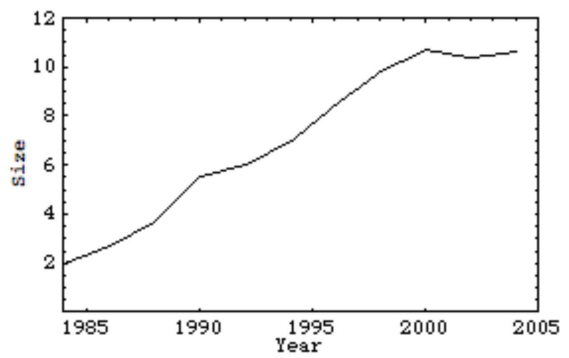

Figure 5. Evolution of average component size for a six-year sliding window.

Mature collaboration networks are dominated by a "giant component" which encompasses most authors $(80 \%-90 \%)$ in the community [9]. Figure 6 shows the size of the giant component as a percentage of the total network size. Clearly, the size of the giant component remains quite small. The declines in the size of the giant component are not due to the giant component shrinking, but rather the size of the total network increasing. The normalized disconnected diameter (NDD) of the network, a metric developed by the authors of this paper, is shown in Fig. 7. The NDD measures how closely the members of the community are connected. An NDD of one indicates that the network is completely disconnected (or each node is maximally separated from the others), consisting only of single nodes with no connections; an NDD of zero indicates that each node has a connection to every other node (or each node is minimally separated from the others). The NDD metric is an extension of the standard network diameter [14] which in our view does not adequately deal with disconnected networks of the type studied here. (A full analysis of the properties and uses of the NDD metric will appear elsewhere.) The NDD for the Hilton Head community remains quite high, indicating that the community is still quite disconnected- most researchers are not connected to one another.

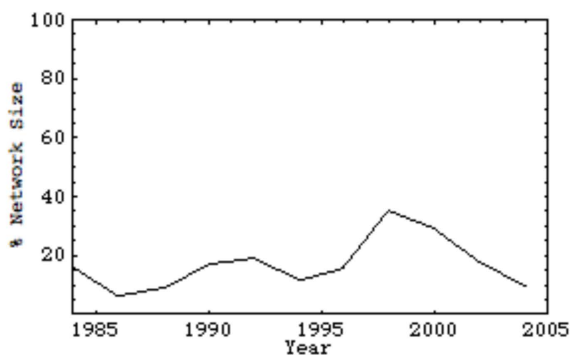

Figure 6. Evolution of the size of the giant component as a percentage of total network size for a six-year sliding window.

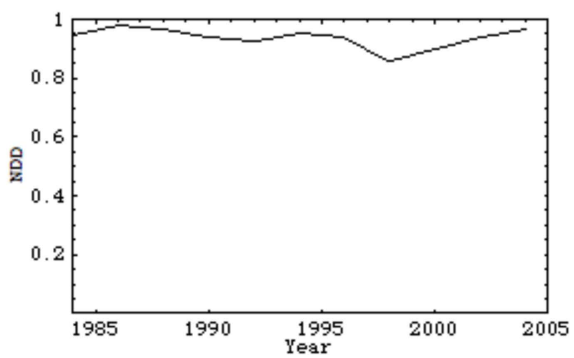

Figure 7. Evolution of the normalized disconnected diameter of the network for a six-year sliding window.

An important role that a researcher can play in the network is that of a "connecter". Connectors are those researchers with the greatest "betweenness" scores. The betweenness score for each author is the number of shortest paths between pairs of authors that the author lies on. These researchers are important because they have the most influence on the network at large in terms of information flow. Table 2 shows the top five betweenness authors for the past six meeting years. The most interesting authors in this table are those that are not also network hubs. Hubs have high betweenness values because they have a large number of collaborators. Those authors which are connectors but have a relatively low number of collaborators are likely to be connectors between separate sub-communities of researchers, such as Mark Sheplak in 2002, for example. Interestingly, at least between 1984 and 2004, the betweenness peaked in 2000 with significantly higher values than were seen before and after that time, which roughly corresponds with the peak in the size of the giant component seen in Fig. 6.

In order to illustrate the importance of authors with high betweenness (but not being network hubs), Fig. 8 shows the local network around Mark Sheplak in 2002, who had a betweenness score of 6411 at that time. It is clear from the figure that though Sheplak had relatively few direct connections (9), he played and extremely important role in this cluster, providing a path between the two sub-communities visible in the figure. Sheplak is the single connector in this cluster, if he were not present, there would be no path between the two sub-communities. 


\begin{tabular}{|c|c|c|}
\hline Year & Author & Belweenness \\
\hline \multirow[t]{5}{*}{2004} & Thomas W. Kenny & 5250 \\
\hline & David J. Becbe & 2184 \\
\hline & Martin A. Schmidt & 1924 \\
\hline & Roger T. Howe & 1922 \\
\hline & Albert P. Pisano & 1800 \\
\hline \multirow[t]{5}{*}{2002} & Thomas W. Kenny & 11193 \\
\hline & James H. Smith & 6496 \\
\hline & Mark Sheplak & 6411 \\
\hline & Roger T. Howe & 5694 \\
\hline & Antonio J. Ricco & 5054 \\
\hline \multirow[t]{5}{*}{2000} & Thomas W. Kenny & 28071 \\
\hline & Roger T. Howe & 14524 \\
\hline & Stephen D. Senturia & 12463 \\
\hline & Yiching A. Liang & 11249 \\
\hline & John R, Gilbert & 11070 \\
\hline \multirow[t]{5}{*}{1998} & Stephen D. Senturia & 23990 \\
\hline & John R. Gilbert & 18587 \\
\hline & James H. Smith & 18271 \\
\hline & Thomas W. Kenny & 17447 \\
\hline & Roger T. Howe & 12896 \\
\hline \multirow[t]{5}{*}{1996} & William J, Kaiser & 3816 \\
\hline & Stephen D. Senturia & 3413 \\
\hline & Khalil Najafi & 2758 \\
\hline & Thomas W. Kenny & 2614 \\
\hline & G. K. Ananthasuresh & 2553 \\
\hline \multirow[t]{5}{*}{1994} & Stephen D. Senturia & 1130 \\
\hline & Mchran Mchregany & 906 \\
\hline & Khalil Najafi & 823 \\
\hline & Kensall D. Wise & 736 \\
\hline & Gregory C. Frye & 428 \\
\hline
\end{tabular}

Table 1. Top five betweenness authors for meeting years of 1994 through 2004.

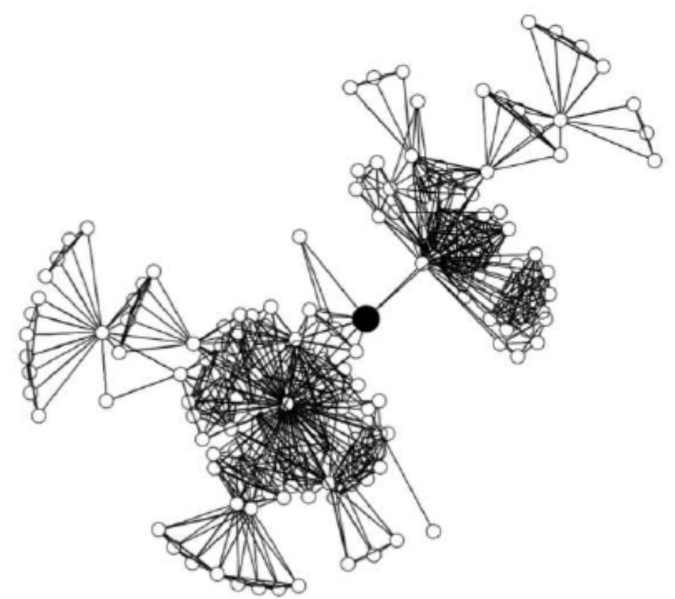

Figure 8. The local network centered around Mark Sheplak (large black circle) in 2002 with a sliding window of six-years.

\section{CONCLUSION}

The Hilton Head workshop research community has undergone great change over the past twenty years. Starting with 37 founders, the community is now made up of nearly one thousand authors. Though the community has grown significantly, it is still quite disconnected when compared to other research communities. There are clear indications that the community is maturing. A distinct group of hub authors has emerged, a property of complex networks. Authors are collaborating with more authors than would be explained simply by an increase in the average number of authors per paper. This has caused the average component size to increase significantly. The continuation of these trends will cause the giant component to grow in size, connecting more authors to one another and decreasing the normalized disconnected diameter. As the community matures, the productivity of its members should increase as it provides a better environment for collaboration and communication.

A limitation of social network analysis of communities of this type is that the membership is controlled artificially in that papers and therefore authors are reviewed before acceptance. Therefore, growth in the field may not be directly evident in the social network. This is not likely to have any great effect on our conclusions however, because such changes will be indirectly evident. As the community grows and the number of papers submitted increases, conference organizers will in-turn increase the size of the conference, accepting more papers.

In contrast to our sliding window analysis, a classical cumulative analysis would have provided some different conclusions. The giant component, for example, would have been significantly larger by 2004 (70\% of network size), which in turn would cause the NDD to be lower, $(0.48)$. We argue though, that such observations would not be valid, as they include collaborations from twenty years in the past.

One limitation of this work is that it includes the collaborations of only one workshop in a larger field. There are likely many collaborations between the authors that are not represented in this workshop. As an analysis of this single workshop though, it still provides interesting insight.

\section{REFERENCES}

[1] S. Lee and B. Bozeman, "The Impact of Research Collaboration on Scientific Productivity", Social Studies of Science, 35, pp. 673-702, (2005).

[2] D.J. de Solla Price, "Networks of scientific papers", Science, 149, pp. 510-515, (1965).

[3] V.Batagelj and A. Mrvar, "Some analyses of Erdös collaboration graph", Social Networks, 22, pp. 173-186, (2000).

[4] P. Erdös and A. Rényi, "On random graphs", Publicationes Mathematicae, 6, pp. 290-297, (1959).

[5] A.L. Barabási and E. Bonabeau, "Scale-free networks", Scientific American, 288, pp. 60-69, (2003).

[6] D.J. Watts and S.H. Strogatz, "Collective dynamics of "smallworld" networks", Nature, 393, pp. 440-442, (1998).

[7] J. Guare, "Six Degrees of Separation", Vintage Books, (1990).

[8] A.L. Barabási, H. Jeong, R. Ravasz, Z. Néda, A. Schubert, and T. Vicsek, "Evolution of the social network of scientific collaborations", Physica A, 311, pp. 590-614, (2002).

[9] M.E.J. Newman, "Coauthorship networks and patterns of scientific collaboration", Proceedings of the National Academy of Science, USA, 101, pp. 5200-5205, (2004).

[10] M.E.J. Newman, "Who is the best connected scientist? a study of scientific coauthorship networks", In H. Frauenfelder E. Ben-Naim and Z. Toroczkai, editors, Complex Networks, pp. 337-370. Springer, (2004).

[11] J.W. Grossman, "The evolution of the mathematical research collaboration graph", Congressus Numerantium, 158, pp. 202212, (2002).

[12] M.A. Nascimento, J. Sander, and J. Pound, "Analysis of sigmod's co-authorship graph", SIGMOD Record, 32, pp. 8$10,(2003)$.

[13] R. Albert and A.-L. Barabási, "Statistical mechanics of complex networks", Review of Modern Physics, 74, pp. 4797 (2002).

[14] S.N. Dorogovtsev and J.F.F. Mendes, "Evolution of networks", Advances in Physics, 51, pp. 1079-1187, (2002).

[15] E.M. Jin, M. Girvan, and M.E.J. Newman, "The structure of growing social networks", Physical Review E, 64, pp. 381-399, (2001). 